GRASAS Y ACEITES, 63 (4),

OCTUBRE-DICIEMBRE, 373-382, 2012,

ISSN: 0017-3495

DOI: $10.3989 /$ gya.035512

\title{
El metabolismo del colesterol: cada vez más complejo
}

\author{
Por J. Sanhueza ${ }^{1, *}$, R. Valenzuela ${ }^{2}$ y A. Valenzuela ${ }^{1,3}$ \\ ${ }^{1}$ Laboratorio de Lípidos y Antioxidantes, Instituto de Nutrición y Tecnología de los Alimentos, \\ Universidad de Chile \\ ${ }^{2}$ Facultad de Medicina, Universidad de Chile \\ ${ }^{3}$ Facultad de Medicina, Universidad de los Andes. Santiago de Chile \\ *Autor para la correspondencia: jsanhueza @ inta.uchile.cl
}

\section{RESUMEN} plejo.

El metabolismo del colesterol: cada vez más com-

El colesterol es una molécula importante; es necesario para la biosíntesis de hormonas esteroideas, de sales biliares y para mantener la estabilidad de las membranas biológicas en células animales. Sin embargo, su exceso es negativo y es responsable de generar enfermedades que comprometen al corazón, al cerebro o que generan algunos tipos de cáncer. Por estos motivos, los niveles de colesterol celulares deben estar finamente regulados, y para ello, participan una infinidad de mecanismos que comprometen al organismo como un todo. Estos mecanismos deben comenzar a operar en forma eficiente desde la ingesta de colesterol dietario para su incorporación al enterocito, donde están implicados transportadores de tipo ABC y NCP1, los motivos estructurales PDZ, entre otras. También debe estar adecuadamente regulado el colesterol circulante y una vez en el interior del organismo, debería establecerse una perfecta armonía entre la incorporación del colesterol a los diversos tejidos, con su utilización metabólica, la generación de reservas de colesterol en los mismos y la síntesis de este lípido. Desde esta perspectiva, la presente revisión ofrece una visión general de los mecanismos moleculares que permiten la regulación de los niveles de colesterol a nivel extra e intra celular.

PALABRAS CLAVE: Colesterol - Metabolismo extraceIular - Metabolismo intracelular - Transporte.

\section{SUMMARY}

\section{Cholesterol metabolism: increasingly complex.}

Cholesterol is an important molecule; it is necessary for the biosynthesis of steroidal hormones, bile salts and to maintain the stability of biological membranes in animal cells. However, its excess is negative and is responsible for the development of many diseases involving the heart and brain, or in the generation of some types of cancer. For these reasons, the cellular cholesterol levels must be finely regulated and therefore, an infinite number of mechanisms participate in this regulation, which undertake the organism as a whole. These mechanisms should begin to operate efficiently from the intake of cholesterol from the diet, its incorporation into the enterocyte, where are involved carriers such as ABC and NCP1 transporters, PDZ structural motif, to name a few. It is also necessary an adequate regulation of circulating cholesterol and once inside the body, there should be a perfect harmony between the addition of cholesterol to various tissues, its metabolic use, the mechanisms of its tissue deposition, and the synthesis of this lipid. From this perspective, this review offers a general view of the molecular mechanisms that allow the regulation of extra and intracellular cholesterol levels.

KEY-WORDS: Cholesterol - Extracellular metabolism Intracellular metabolism - Transport.

\section{INTRODUCCIÓN}

Desde su descubrimiento en 1769 por el fisiólogo Francoise Poulletier de la Salle, el colesterol ha sido motivo de numerosos estudios, investigaciones y publicaciones (Olson, 1998). Hoy sabemos más sobre su digestión, biosíntesis, sus importantes funciones bioquímicas, su regulación y su participación en el origen de numerosas enfermedades (cardio y cerebro vasculares, neurológicas, inflamatorias, etc). Sin embargo, solo en forma más reciente se han descubierto, y entendido, los intrincados mecanismos moleculares que regulan su transporte fuera y dentro de las células, los mecanismos regulatorios en los cuales interviene y las numerosas moléculas que además del colesterol intervienen en estos procesos. El propósito de esta revisión es analizar lo que hoy día sabemos sobre estos últimos aspectos.

\section{DIGESTION Y ABSORCION DEL COLESTEROL}

El $90-95 \%$ del colesterol que consumimos en la dieta está esterificado, por lo cual la enzima colesterol esterasa de origen pancreático lo libera en el intestino delgado (Howles et al., 1996). Una vez incorporado a las micelas mixtas, es transferido activamente a los enterocitos a través de la proteína transportadora Niemann-Pick C1 tipo 1 (NPC1) que se ubica en la membrana apical de los enterocitos y también en la membrana canalicular de los hepa- 
tocitos, donde interviene en el transporte reverso del colesterol (tejidos periféricos $\rightarrow$ hígado) (TRC) (Altmann et al., 2004). La mayor parte del colesterol es reesterificado en el retículo endoplasmático (RE) del enterocito por la enzima acil-CoA colesterol aciltransferasa 2 (ACAT2), para ser incorporado a los quilomicrones nacientes junto con los triglicéridos (Clark and Tercyak, 1984). La ACAT2 es relativamente saturable en los humanos, por lo cual frente a una ingesta alta de colesterol no todo se esterifica (Iqbal and Hussain, 2009). El colesterol no esterificado puede seguir tres caminos: la mayor parte es devuelta al lumen intestinal, junto con otros esteroles (ej, fitoesteroles) por los transportadores ABCG5/G8 (ABC: ATP Binding Cassette) (Ikonen, 2008); otra parte es transportada a la membrana basolateral del enterocito para la biogénesis de las HDL de origen intestinal, proceso que realiza el transportador ABCA1 (Tamehiro et al., 2008); y una fracción menor se incorpora como tal (no esterificado) intercalándose en los fosfolípidos que forman el quilomicrón, el que además incorpora la apoproteína B48 (ApoB48) (Hassan et al., 2007; Ikonen 2008; Weinstein et al., 2010). El transporte del colesterol por ABCA1 es regulado tanto en la transcripción como en la modificación postranscripcional del transportador (Okuhira et al., 2005), con lo cual es posible aumentar o disminuir su actividad. En la regulación postranscripcional participa una proteína ( $\beta$-sintrofina) la que al unirse al motivo estructural PDZ estabiliza al transportador y facilita la transferencia del colesterol a la HDL discoidal, la cual además incorpora la apoproteína A1 (ApoA1), asegurando así un mejor transporte del colesterol en exceso, el que vía HDL va al hígado (Okuhira et al., 2005; Okuhira et al., 2010). A este proceso se le conoce como transporte transintestinal del colesterol y da cuenta del $30 \%$ del TRC (van der Velde et al., 2010). El colesterol que no participa en alguna de las tres vías (transporte por ABCG5/G8, ABCA1 y quilomicrón) se puede oxidar formando oxisteroles. Estos metabolitos son agonistas del receptor $X$ hepático (LXR) (que también se expresa en los enterocitos) cuya activación regula positivamente la expresión de los transportadores $\mathrm{ABC}$, asegurando así el transporte del colesterol fuera del enterocito. De esta forma los LXR actuarían como "sensores" del exceso de colesterol intraenterocítico (Janowski et al., 1999; Zhao and Dahlman-Wright, 2010). La figura 1 muestra en forma esquemática el proceso de digestión, absorción y los destinos iniciales del colesterol.

\section{TRANSPORTE PLASMATICO DEL COLESTEROL}

Una vez formados los quilomicrones en el RE del enterocito, estos viajan a través de vesículas recubiertas de COPII a la membrana basolateral de

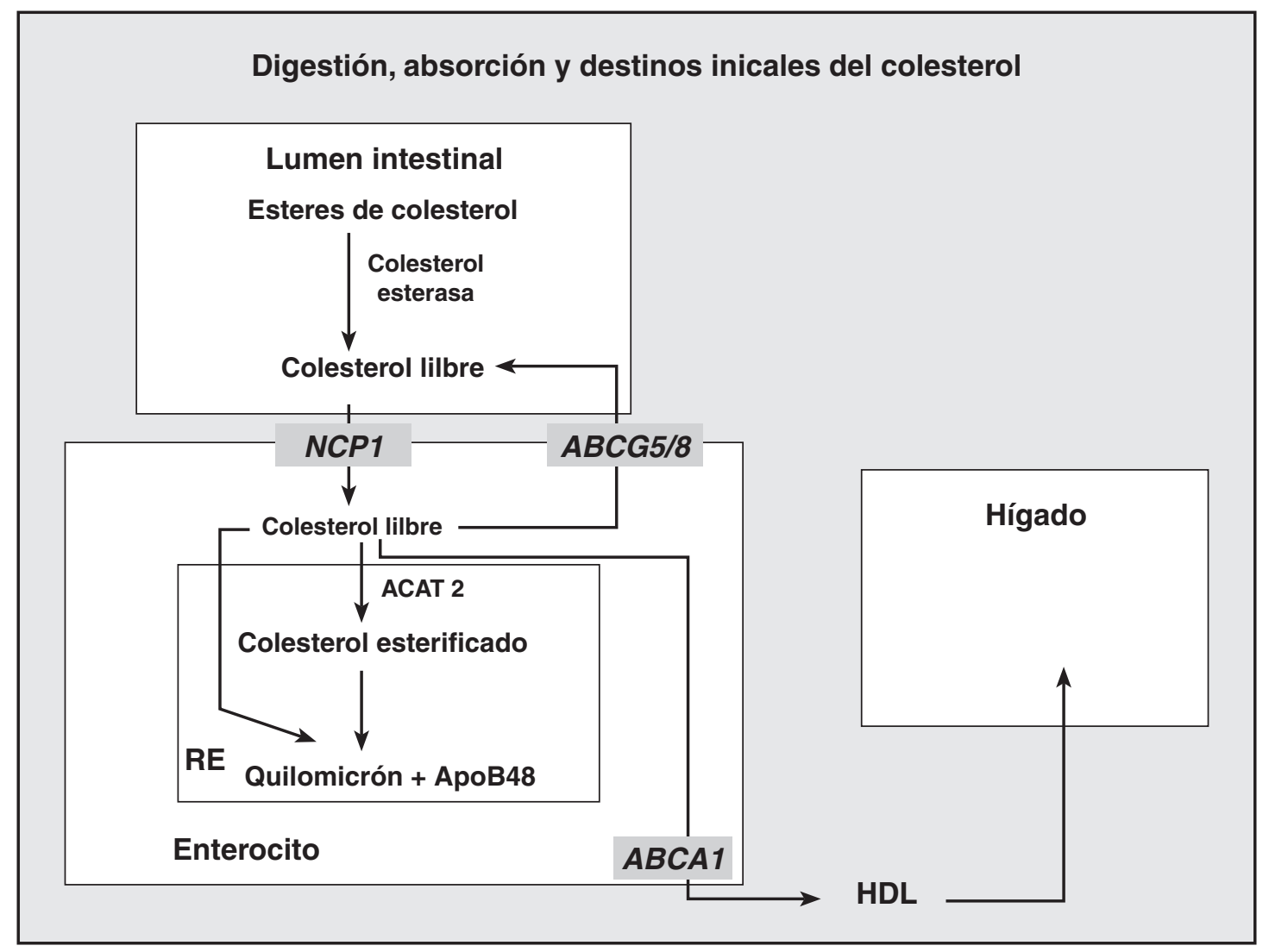

Figura 1

Esquema del proceso de digestión, absorción y destinos iniciales del colesterol. 
estas células (Nickel et al., 1998). Así son vaciados al sistema linfático y posteriormente al sistema vascular, vía el conducto torácico (Ikonen, 2008). En los capilares que irrigan a los tejidos (principalmente adiposo y muscular) se encuentra la enzima lipoproteína lipasa (LPL), la que degrada los triglicéridos del quilomicrón enriqueciéndolo en colesterol (Santamarina-Fojo and Dugi, 1994). Los remanentes de quilomicrones son internalizados en las células hepáticas y su contenido liberado por la actividad lisosomal de estas células (López-Soldado et al., 2007; López-Soldado et al., 2009).

Las células hepáticas secretan constantemente VLDL las que en el plasma se van transformando sucesivamente hasta LDL (Lewis, 1997). Estas lipoproteínas son reconocidas por las células que poseen receptores de LDL (LDLr). Estos receptores reconocen lipoproteínas que contienen $A p o B$ (48 y 100) o ApoE, por lo cual pueden reconocer a quilomicrones, VLDL, IDL y LDL, siendo mucho más específicos para las LDL (Rohlmann et al., 1998; Lin et al., 2004). Las vesículas de endocitosis, recubiertas de clatrinas y que llevan receptores y LDL, viajan hasta endosomas tardíos, los que al transformarse en lisosomas degradan a las LDL a través de las lipasas ácidas (Lin et al., 2004). Los ésteres del colesterol son hidrolizados por una colesterol esterasa similar a la pancreática. Antes que ocurra este proceso hidrolítico, los receptores se liberan del endosoma y son reciclados (Laatsch et al., 2012). El colesterol puede abandonar la vesícula lisosomal a través de los transportadores NPC1 y NPC2 (más ubicuos que los NPC1) que se encargan de exportar el colesterol libre hacia sus destinos: membrana plasmática, RE, endosomas de reciclaje y mitocondrias (Ioannou, 2001; Storch and $\mathrm{Xu}, 2009$ ).

\section{REGULACION INTRACELULAR DEL COLESTEROL}

La síntesis, captación y procesamiento intracelular del colesterol son regulados principalmente por dos receptores nucleares: la proteína de unión a elementos reguladores de esteroles (SREBP) y el LXR (Calkin and Tontonoz, 2010; Sato, 2010). SREBP estimula la expresión de genes que promueven el aumento del colesterol celular cuando este está bajo, mientras que LXR estimula el transporte reverso de colesterol, disminuyendo sus niveles intracelulares en las células periféricas (Tontonoz and Mangelsdorf, 2003). La regulación precisa del colesterol intracelular requiere la participación activa de varios componentes de la membrana del RE. Estos componentes pueden permanecer o desaparecer de la membrana de acuerdo a los niveles intracelulares de colesterol. Cuando el nivel intracelular de colesterol es bajo, se produce una interacción entre SREBP y la chaperona SCAP formándose el dímero SREBP-SCAP. Ambas proteínas se trasladan al Golgi donde SREBP es activado por proteasas. Una vez activado SREBP este se traslada al núcleo, donde induce la expresión de la enzima HMG-CoA red, de LDLr y de la chaperona INSIG (proveniente de genes que también son inducidos por la insulina) la que promueve la degradación de la enzima HMG-CoA red. Si la chaperona INSIG no se une al dímero SREBP-SCAP, es degradada en el proteosoma y por lo tanto no esta disponible para permitir la degradación de HMGCoA red, lo que solo ocurre cuando INSIG se une a SREBP-SCAP. En esta condición la célula puede captar colesterol (debido a la síntesis de LDLr) y al mismo tiempo biosintetizarlo (debido a la mayor disponibilidad de HMG-CoA red), con lo cual los niveles de colesterol aumentan. Cuando el colesterol intracelular alcanza determinada concentración, el exceso que no se utiliza se une a SREBP, el que a su vez está unido a SCAP. Al conjunto molecular colesterol-SREBP-SCAP se le une INSIG, lo que permite su estabilización evitando la acción del proteosoma, favoreciendo la degradación de la enzima HMG-CoA red, con lo cual bajan los niveles intracelulares de colesterol, iniciándose así un nuevo ciclo recíproco de regulación (Goldstein et al., 2006; Gong et al., 2006). La figura 2 muestra en forma esquemática el control intracelular del metabolismo del colesterol.

\section{EL FLUJO INTRACELULAR DEL COLESTEROL}

\subsection{Organización celular y el colesterol}

El colesterol posee la propiedad de ser "molécula ordenadora" de los lípidos (fosfolípidos) en las membranas celulares (Ikonen, 2008). Debido a la rigidez del esqueleto esterol que posee el colesterol, este se ubica principalmente entre cadenas hidrocarbonadas saturadas, ya que son más largas y más inflexibles comparadas con las cadenas insaturadas (Simons and Vaz, 2004). El orden lateral que genera el colesterol entre los lípidos vecinos, modifica las propiedades biofísicas de la membrana, reduciendo la fluidez, disminuyendo la movilidad lateral de las proteínas y controlando la permeabilidad a moléculas polares (Martinez-Seara et al., 2010). La razón colesterol/proteínas en las membranas de células de mamíferos varía notablemente. El esterol es distribuido heterogéneamente entre las distintas membranas celulares siendo más abundante en la membrana plasmática y del Golgi (Waugh et al., 2011). En la membrana plasmática el colesterol constituye alrededor del $80-90 \%$ del colesterol celular total y entre el 20-25\% del total de moléculas lipídicas (Ikonen, 2008). El colesterol es abundante, especialmente en el trans-Golgi y en los compartimientos endocíticos de reciclaje (Waugh et al., 2011). En contraste, tanto en la mitocondria como en el RE los niveles de colesterol son muy bajos. El colesterol en el RE da cuenta sólo del $1 \%$ del colesterol total de la célula (Mukherjee et al., 1998). La distribución asimétrica del colesterol en membrana plasmática y 


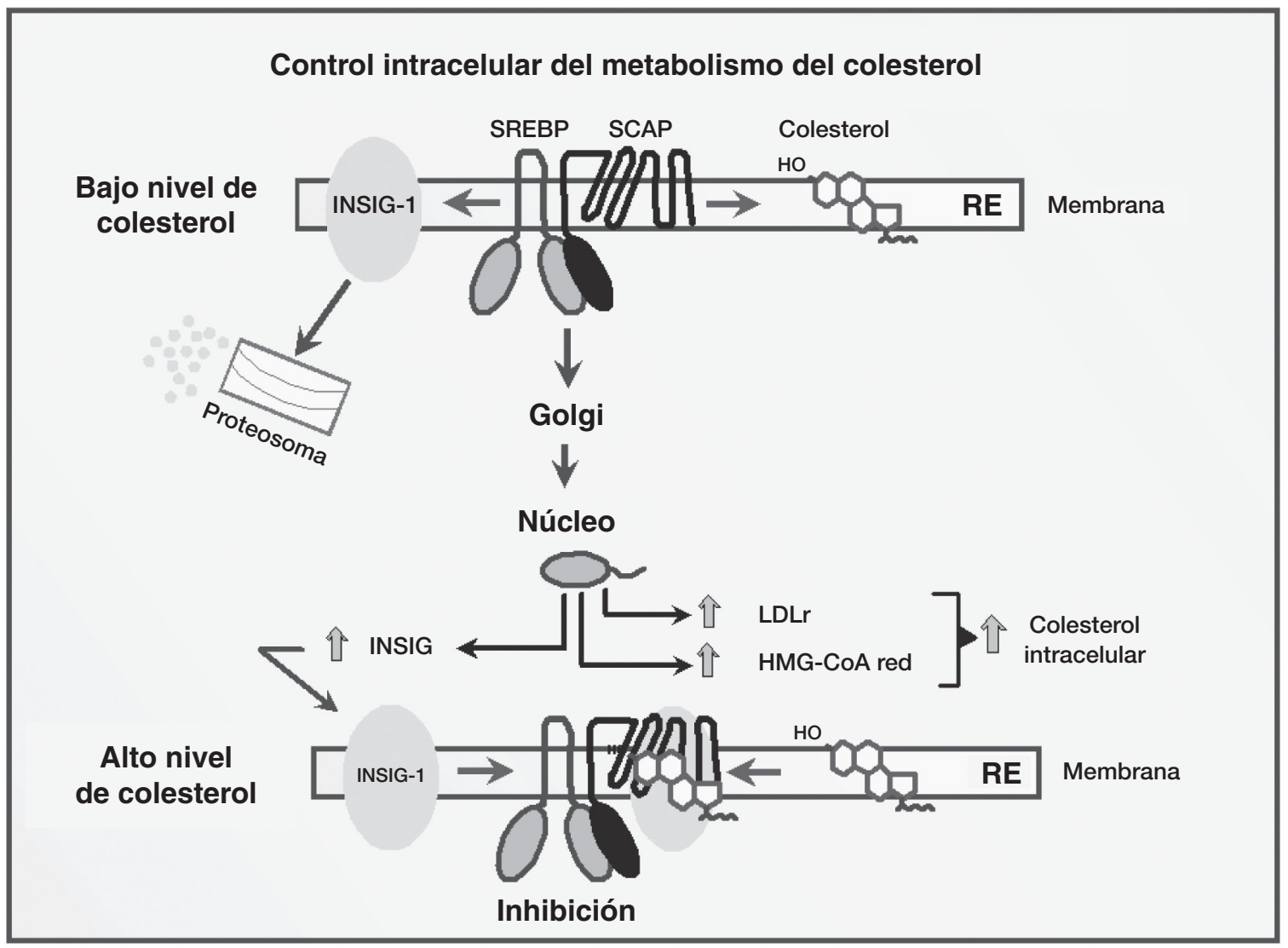

Figura 2

Regulación recíproca de los niveles intracelulares de colesterol. Cuando los niveles de colesterol están disminuidos, el sistema trabaja de manera que este se sintetice, esto implica que Insig 1 se degrada y el conjunto SREBP/SCAP migra al Golgi donde SREBP es activado para ir al núcleo e inducir un aumento en los niveles de HMG-CoA red y LDLr, lo que se traduce en un aumento de colesterol intracelular. Además, en el núcleo SREBP produce un aumento de Insig 1. Una vez elevados los niveles de colesterol, Insig 1 se une al complejo SREBP/SCAP de manera que ahora Insig 1 puede promover la degradación de SREBP, cuyo efecto será la disminución de los niveles de colesterol, hasta que se reinicie un nuevo ciclo. Modificada de Gong et al., 2006

del RE, es constante. La concentración de colesterol en el RE controla varias funciones relacionadas con el transporte RE $\rightarrow$ Golgi, tales como la propia homeostásis del esterol, la actividad de las proteínas residentes en el RE y la salida de proteínas de membrana sintetizadas en el RE (loannou, 2001; Feng et al., 2003; Goldstein et al., 2006).

\subsection{Colesterol y caveolas}

Las caveolas son invaginaciones (50-100 nm) de la membrana celular. Su forma de tipo vesicular y su localización en la superficie han sugerido su participación en eventos celulares como la endocitosis, la transcitosis y/o el tráfico vesicular y el ensamblaje de complejos que participan en vías de señalización (Pilch et al., 2011). Las caveolas constituyen el $50 \%$ de la superficie de los adipocitos, lo que ha sugerido que estas estructuras tendrían por función modular el movimiento de lípidos, ya que están particularmente enriquecidas en colesterol y esfingomielina (Thorn et al., 2003; Ortegren et al., 2004). Los elevados niveles de colesterol en las caveolas de la membrana plasmática son necesarios para que estas adquieran su ca- racterística forma de cueva (de ahí su nombre). Si las células se tratan con un antibiótico (nistatina) que secuestra colesterol, las caveolas se transforman en estructuras planas (Rothberg et al., 1992). En cultivo de fibroblastos se ha mostrado que la velocidad de eflujo de colesterol libre y los niveles de mRNA de la principal proteína constituyente de las caveolas, la caveolina 1 (Cav-1), son proporcionales al contenido de colesterol libre en las caveolas, de modo que cuando se bloquea la expresión de Cav-1, se inhibe el eflujo de colesterol desde la membrana (Fielding et al., 1997; Nabi and Le, 2003). Adicionalmente, el contenido de colesterol en la membrana plasmática es esencial para otras funciones de las caveolas, ya que la depleción de colesterol en la membrana, inhibe la pinocitocis y dispersa proteínas de anclaje, lo que lleva a una interrupción de las cascadas de transducción de señales (Hannan and Edidin, 1996; Shaul, 2003).

\subsection{Tráfico intracelular del colesterol}

Los procesos bioquímicos en los que interviene el colesterol ocurren en distintos organelos celula- 
res y por lo tanto su movimiento hacia ellos es una forma de regular su metabolismo (Girard et al., 2011). Una vez sintetizado el colesterol en el RE, este se traslada rápidamente a las diferentes membranas, incluida la membrana plasmática, evitando el Golgi, en un proceso que es dependiente de energía (loannou, 2001). Este transporte ocurre a través de vesículas, las que se movilizan a través del citoesqueleto (microtúbulos) (Damm et al., 2005). Además, existe un transporte de colesterol no mediado por vesículas, donde proteínas citosólicas de transferencia lipídica realizan el intercambio directo membrana-membrana (Hanada et al., 2003). Estas proteínas poseen un dominio de unión a colesterol y diferentes dominios de unión a membranas donantes y aceptoras (Hanada et al., 2003). El colesterol que no es transportado a membranas, es esterificado a través de la ACAT2, en forma similar al proceso que ocurre en los enterocitos (Ikonen, 2008). El colesterol esterificado es almacenado en gotas lipídicas que sobresalen del RE (Olofsson et al., 2009).

\subsection{El colesterol en gotas lipídicas}

Para la célula (al igual que para el organismo) existen períodos de abundancia y otros de escasez, por lo tanto, poseer un mecanismo de almacenamiento de energía y/o de moléculas claves, puede ser una ventaja evolutiva (Otsuka, 2009). El colesterol esterificado se acumula, principalmente en los adipocitos, en grandes gotas lipídicas y de tamaño muy variable (diámetro entre 1 y 100nm) (Farese and Walther, 2009). Estas consisten en una monocapa de fosfolípidos que rodea un centro de lípidos neutros como el colesterol esterificado y los triglicéridos, además de numerosas proteínas en su superficie (perilipinas), de manera similar a las lipoproteínas plasmáticas (Farese and Walther, 2009; Valenzuela and Sanhueza, 2009). El metabolismo en estas gotas lipídicas es muy dinámico y a partir de ellas se ejercen muchos mecanismos regulatorios. Por ejemplo, se les atribuye una función amortiguadora del exceso de colesterol libre, el que se almacenaría en forma de colesterol esterificado cuando aumenta su nivel intracelular, una función relevante ya que el exceso de colesterol es tóxico para la célula (Jaureguiberry et al., 2010). Se ha propuesto, además, que el colesterol podría participar en la regulación del ciclo celular, debido a que se ha observado acumulación de ciclinas sobre la superficie de las gotas lipídicas (Dong et al., 2010). La caveolina, además de estar asociada a las caveolas, también se asocia con las gotas lipídicas. Cuando aumenta el colesterol, se produce un tráfico de caveolinas desde la membrana plasmática hacia las gotas lipídicas, donde estas proteínas jugarían un rol importante en mantener el nivel de colesterol libre (Fielding et al., 1997). Además, el transportador de colesterol ABCG1, también regularía el transporte de triglicéridos en las gotas lipídicas (Ikonen, 2008).

\subsection{Transporte de colesterol a la mitocondria en la esteroidogénesis}

Los principales órganos productores de hormonas esteroideas son los testículos, los ovarios y la glándula adrenal. La síntesis de hormonas esteroideas es regulada por hormonas tróficas como la hormona adrenocorticotrófica (ACTH) en las células de la corteza adrenal y la hormona luteinizante (LH) en las células de Leydig de los testículos y células de la teca en los ovarios (Liu et al., 2003). El principal punto de control de la estimulación esteroidogénica por hormonas peptídicas y AMPc, es el primer paso en la vía biosintética, donde el colesterol es convertido en pregnenolona por la enzima citocromo P-450scc (del inglés P-450 side-change cleavage) (Liu et al., 2003). Esta enzima se encuentra en la membrana interna de la mitocondria (MIM) (Jefcoate, 2002). StAR, es una proteína que se ubica en la membrana externa mitocondrial (MEM) y que está involucrada en la modulación del transporte de colesterol desde el citosol hasta la MIM (Liu et al., 2003). Así, cuando se requiere una respuesta esteroidogénica aguda, aumenta rápidamente la síntesis de la proteína transportadora StAR. El transporte del colesterol a través de las membranas de la mitocondria lo realizaría un complejo proteico en el cual, además de la proteína StAR también habrían otros componentes, como el receptor periférico de benzodiacepinas (PBR), la proteína asociada a PBR (PAP7) y una proteína kinasa, dependiente de AMPc (PKA) (Rone et al., 2009). Este complejo respondería a hormonas las que mediante señales de AMPc regularían el transporte de colesterol a través de la MEM para la síntesis de hormonas esteroideas en la MIM (Liu et al., 2003). Para la regulación del transporte de colesterol hasta la MIM, PAP7 se uniría a la subunidad regulatoria de la PKA, permitiendo una activación de StAR mediante fosforilación, resultando en un rápido traspaso de colesterol desde StAR a PBR, trasladando así el colesterol desde la MEM hacía la MIM, donde la enzima P450scc lo transformaría en pregnenolona, para continuar posteriormente la síntesis de hormonas esteroideas en el RE (Hauet et al., 2002).

\section{CITOTOXICIDAD DEL COLESTEROL}

Los macrófagos acumulan colesterol libre en las lesiones ateroescleróticas avanzadas, generando un circulo maligno, ya que se produciría un proceso inflamatorio, induciéndose apoptosis y la progresión de la lesión (Feng et al., 2003). Cuando el colesterol intracelular sobrepasa los niveles de colesterol suficientes para cumplir con sus funciones, este se transforma en un componente tóxico para la célula. Se han propuesto varios mecanismos de citotoxicidad por colesterol, los que incluyen la formación de cristales de colesterol, la inducción de respuesta a proteínas mal plegadas (URP), la formación de oxisteroles, la disrupción de dominios 
de membrana necesarios para la transducción de señales y la activación de vías intrínsecas y extrínsecas de apoptosis (Lordan et al., 2009). La acumulación experimental de colesterol intracelular produce externalización de la fosfatidilserina (transferencia desde la cara interna de la membrana a la externa) y fragmentación del DNA, eventos que evidencian una mayor apoptosis (Yao and Tabas, 2000). El aumento no controlado de colesterol en el RE activa la liberación de calcio al citoplasma, lo cual constituye una respuesta pro apoptósica que activaría a URP, iniciando la expresión de proteínas efectoras de apoptosis (Feng et al., 2003).

\section{EFLUJO DEL COLESTEROL}

El eflujo de colesterol desde los macrófagos es un evento clave que permite evitar las lesiones ateroscleróticas y forma parte inicial del TRC. En las células espumosas, el colesterol se encuentra mayormente en gotas lipídicas, en forma de esteres de colesterol, y su hidrólisis aumenta la velocidad de eflujo (Attie, 2007; Meurs et al., 2010). El descubrimiento que mutaciones en el ABCA1 están asociadas con una deficiencia de HDL, llevó a explicar la función de este transportador, ya que es a través de $A B C 1$ que el colesterol y los fosfolípidos son exportados a apolipoproteinas aceptoras (precursoras de HDL) en la circulación sanguínea, siendo crucial para la formación de las HDL (Attie, 2007). ABCA1, se encuentran en muchos tipos celulares y su actividad es la etapa limitante en la formación y la mantención de los niveles plasmáticos de las HDL. Como ya se mencionó, en el intestino ABCA1 transporta colesterol desde los enterocitos para la formación de un tercio de la HDL plasmática total (Fitzgerald et al., 2010; van der Velde et al., 2010). Por otro lado, en las paredes arteriales, este transportador es importante en la eliminación del exceso de colesterol desde los macrófagos, con lo cual ABCA1 tendría un rol anti-aterogénico (van der Velde et a.l, 2010). Estudios en ratones knock-out (KO) para este transportador han demostrado que en los enterocitos y en los hepatocitos el ABCA1 es clave para mantener los niveles de HDL circulantes. La principal apolipoproteina de las HDL, ApoAI, se ancla a ABCA1 con lo cual la HDL naciente, inicialmente vacía de colesterol, empieza a adquirir desde las células periféricas, fosfolípidos y colesterol que van enriqueciendo y modificando su estructura desde una forma discoidal a una estructura semi-esférica (Meurs et al., 2010; Smith, 2010). ABCA1 se concentra mayoritariamente en microdominios de la membrana plasmática enriquecidos en fosfatidilcolina y en menor proporción de esfingomielina (Hassan et al., 2007). En el proceso de eflujo del colesterol los eventos endocíticos juegan un rol importante, ABCA1 contribuye al eflujo de colesterol desde los endosomas tardíos, proceso que involucra a NPC1. El transportador ABCG1, es altamente expresado en los macrófagos, a diferencia de ABCA1 que es más ubicuo. ABCG1 sinergi- za con ABCA1 en la formación de la HDL madura, cooperando en la incorporación de fosfolípidos a la lipoproteína que solo posee ApoA1 (Chen et al., 2005; Hassan et al., 2007). Esto es apoyado por experimentos in vitro, donde en macrófagos que no expresan ABCA1 y tampoco ABCG1, se produce una completa paralización del eflujo de colesterol. Además, ratones KO para el LDLr, trasplantados con médula ósea de ratones $K O$ para $A B C A 1$ y $A B-$ CG1, y que por lo tanto poseen macrófagos deficiente en ambos transportadores, presentan una extensa infiltración con células espumosas en el miocardio y el bazo, y una aterogénesis avanzada (lkonen, 2008).

EI TRC finaliza en el hígado, donde los hepatocitos captan el colesterol extraído desde los tejidos periféricos, a través de las HDL, mediante la unión de las HDL maduras al receptor scavenger clase $B$ tipo 1 (SRB1). Luego, el colesterol es eliminado por el canalículo biliar a través de los transportadores ABCG5/G8. La expresión de SRB1 se correlaciona con la secreción biliar de colesterol (lkonen, 2008; Jakulj et al., 2010). Adicionalmente, se ha reportado que una proteína similar a la angiopoietina (Angptl4-Tg) funciona como moduladora del metabolismo de las lipoproteínas, debido a que es una molécula reguladora de la LPL plasmática. Angptl4Tg puede procesar a la LPL transformándola en un monómero sin actividad, efecto que también ejerce sobre la lipasa hepática (Lichtenstein et al., 2007; Sukonina et al., 2006). Así, en animales ayunados por 24 horas se observa un aumento de la expresión de Angptl4-Tg, lo que se traduce en una menor actividad hidrolítica de las VLDL por parte de la LPL, en un aumento de los triglicéridos plasmáticos y en un aumento de la expresión de los genes implicados en la síntesis de hepática de colesterol. En estas condiciones, el hígado disminuye la captación de colesterol como consecuencia de la inhibición de la actividad de la LPL y de la actividad de la lipasa hepática. En estas circunstancias, disminuye la transformación de las VLDL a LDL, con lo cual disminuye el aporte de colesterol a las células vía LDL, lo que estimula la biosíntesis endógena de colesterol (Lichtenstein et al., 2007).

\section{LOS COMPONENTES DE LA DIETA EN LA HOMEOSTASIS DEL COLESTEROL}

En la homeostasis del colesterol se requiere de controles a nivel intracelular, a nivel extracelular y en el compartimiento plasmático, siendo el hígado el principal órgano involucrado en la homeostasis de colesterol (Dietschy et al., 1993). Además, se requiere de una eficiente captación del colesterol mediante el LDLr, de liberación de lipoproteínas en la sangre, de almacenamiento en tejidos por acción de esterificación, de degradación, y conversión en ácidos biliares, entre otras tareas (Weber et al., 2004). Actualmente se ha observado que la edad, la raza, o el género, afectan la absorción de colesterol (Martini \& Pallottini 2007), pero además, la in- 
gesta de alimentos y en especial de componentes bioactivos pueden actuar como reguladores del colesterol, ejerciendo efecto en variadas proteínas que modulan la expresión de genes, ya sea relacionados con el metabolismo del colesterol como de otros lípidos, sobre todo, si el efecto es ejercido a través de SREBPs como lo muestra la figura 3.

Los distintos componentes de la dieta pueden afectar la regulación del colesterol. Por ejemplo, la ingesta de carbohidratos, cuya respuesta posprandial típica es el aumento de la insulina, conlleva a la estimulación de la actividad transcripcional de SREBP-2 y SREBP-1c, utilizando la vía de proteínas kinasas activadas por mitógenos (MAPK) (Martini and Pallottini 2007). Por otra parte, se ha observado que con la adición de ácidos grasos omega-3 (eicosapentaenoico, EPA y docosahexaenoico, $\mathrm{DHA}$ ) a enterocitos en cultivo se obtiene un $58 \%$ de reducción de la expresión del gen NPC1L1, mientras que el ácido linoleico, el ácido palmítico y el ácido oleico no tienen efecto sobre la expresión de este gen. Estos resultados explicarían el efecto hipolipidémico de los ácidos grasos omega-3. Una forma de actuar del EPA y del DHA es mediante disminución de la actividad de SREBPs y del dímero conformado por LXR con el receptor retinoico $X$ (RXR) (LXR/RXR) (Alvaro et al., 2010). Otra forma de actuar de estos ácidos grasos es mediante la expresión aumentada del transportador ABCG5/8 y del aumento de la expresión de la enzima 27 colesterol hidroxilasa por inducción del citocromo Cyp27a1 (Kamisako et al., 2012). Un estudio realizado en hamsters demostró que el ácido graso omega-3 docosapentaenoico (DPA) y el DHA, reducen el colesterol total plasmático y el colesterol no HDL (colesterol de LDL y de VLDL) a través de una disminución de la actividades de SREBP2, de la enzima HMG-CoA red y de la enzima CYP7A1 (que forma parte de la familia de citocromos que catabolizan al colesterol) (Chen J, et al., 2012). Sin embargo, el efecto de los ácidos grasos omega-3, según investigaciones recientes, depende de la fuente de origen, ya que se ha demostrado un mayor efecto en la inhibición de la síntesis de colesterol cuando estos ácidos grasos provienen de fosfolípidos marinos, cuya biodisponibilidad es mayor que la de los triglicéridos marinos (Mathews et al., 2002) Es así como el EPA y DHA proveniente de fosfolípidos marinos muestran una mayor efectividad para inhibir las actividades de PPAR $\alpha$, de SREBP 1-c y de SREBP 2, con la consiguiente disminución de la síntesis de HMG-CoA red y de otras enzimas que tienen que ver con la síntesis de colesterol (Burri et al., 2011).

Los fitoestrógenos son compuestos bioactivos vegetales que se pueden unir a receptores de estrógenos e inducir una respuesta estrogénica/antiestrogénica (Kuiper et al., 1998). Así, la ginesteína y la daidzeína, fitoestrógenos que se encuentran en legumbres como la soya, pueden modificar la li-

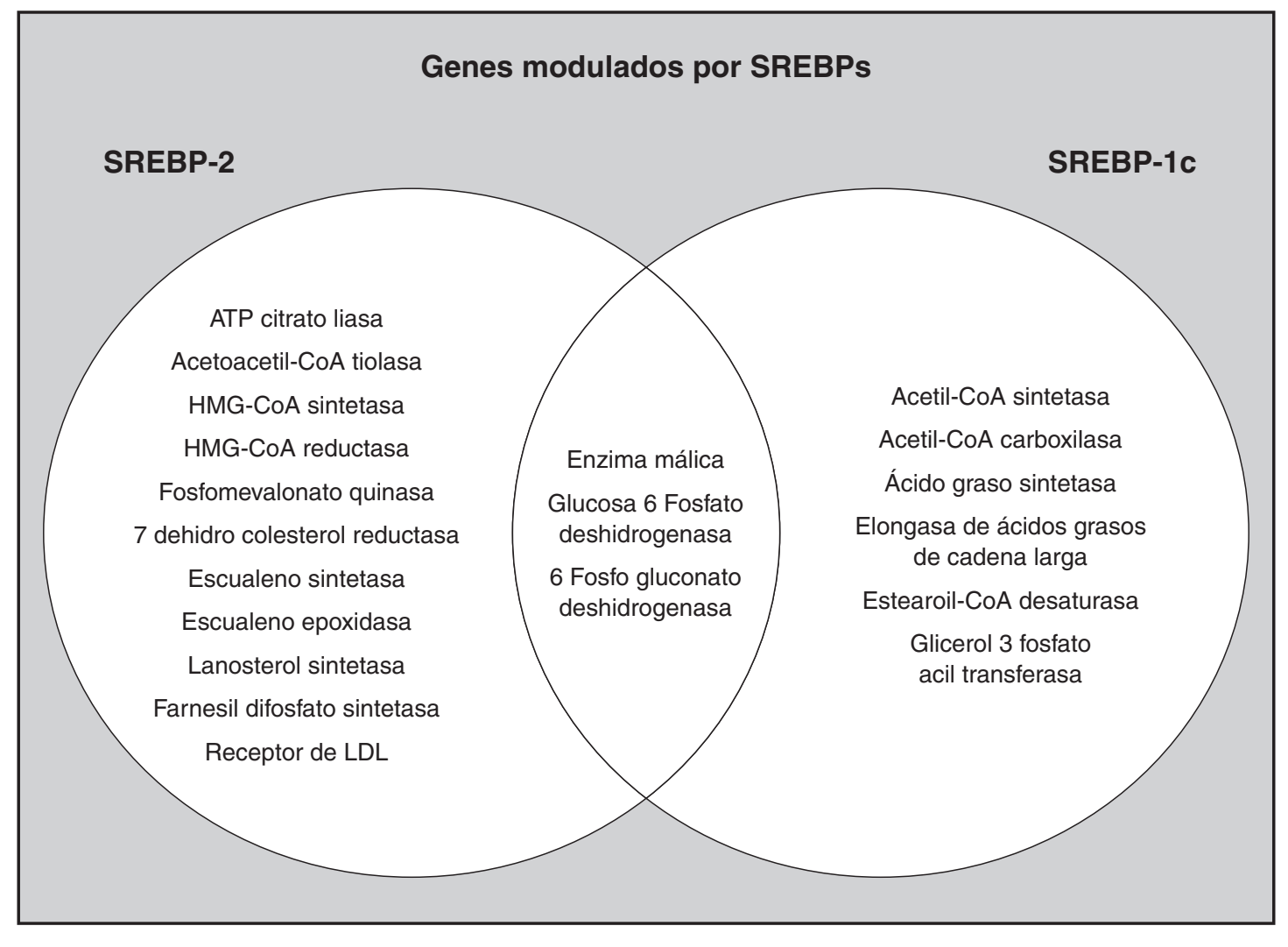

Figura 3

Reguladores del colesterol, efecto ejercido a través de SREBPs. Modificada de Martini C and Pallottini V. 2007 
pogénesis y los niveles de colesterol modulando la expresión de SREBP 1-c, PPAR- $\gamma$ y LXR- $\alpha$ (Ronis et al., 2009). Otros componentes bioactivos de los alimentos pueden reducir los niveles de colesterol dentro de límites saludables, como es el caso del resveratrol y de otros polifenoles (Magyar et al., 2012), o el efecto antiaterogénico del flavonoide quercitrina al actuar como inhibidor de la oxidación de las LDL (Choi et al., 2010). También las catequinas del té son eficientes en disminuir en forma significativa los niveles de colesterol (Kim et al., 2011). Sin embargo, a pesar de los muchos estudios que muestran cambios en el sentido de regular los niveles de colesterol por el efecto de sustancias bioactivas de la dieta, es preciso notar que la respuesta de los individuos a los nutrientes no es común a todas las poblaciones, debido a diferencias polimórficas de varios genes que participan en el metabolismo lipídico, como el del colesterol (Aung et al., 2011; Kotani et al., 2011; Pan et al., 2012), o a modificaciones postranscripcionales de los productos génicos (Medina et al., 2011).

\section{CONCLUSIONES}

Mientras más conocemos sobre el metabolismo del colesterol, más complejo es aún entender las intrincadas interacciones que tiene esta importante molécula. El colesterol es casi la única molécula de nuestro organismo que no se destruye metabólicamente, esto es, o se le utiliza en sus múltiples funciones, o se le debe eliminar. En ambos procesos participan un gran número de moléculas que paulatinamente han sido identificadas y podemos presumir que aún quedan muchas otras por identificar. El conocimiento y la comprensión en detalle del laberinto metabólico en que participa el colesterol, nos permitirá entender mejor las enfermedades asociadas a su desregulación y el desarrollo de intervenciones nutricionales y/o medicamentos que permitan corregir o modificar estas desregulaciones, que en su conjunto causan numerosas enfermedades, entre ellas las cardio y cerebro vasculares, que constituyen hoy día la primera causa de morbi/mortalidad en el mundo occidental.

\section{REFERENCIAS}

Altmann SW, Davis HR Jr, Zhu LJ, Yao X, Hoos LM, Tetzloff G, lyer SP, Maguire M, Golovko A, Zeng M, Wang L, Murgolo N, Graziano MP. 2004. NiemannPick C1 Like 1 protein is critical for intestinal cholesterol absorption. Science. 303, 1201-1204.

Alvaro A, Rosales R, Masana L, Vallvé JC. 2010. Polyunsaturated fatty acids down-regulate in vitro expression of the key intestinal cholesterol absorption protein NPC1L1: no effect of monounsaturated nor saturated fatty acids. J. Nutr. Biochem. 21, 518-525

Attie AD. 2007. ABCA1: at the nexus of cholesterol, HDL and atherosclerosis. Trends Biochem Sci. 32, 172-179.

Aung LH, Yin RX, Wu DF, Li Q, Yan TT, Wang YM, Li H, Wei DX, Shi YL, Yang DZ. 2011. Association of the
TRIB1 tribbles homolog 1 gene rs17321515 A>G polymorphism and serum lipid levels in the Mulao and Han populations. Lipids Health Dis. 10, 230.

Burri L, Berge K, Wibrand K, Berge RK, Barger JL. 2011. Differential effects of krill oil and fish oil on the hepatic transcriptome in mice. Front Genet. 2, 45.

Calkin AC, Tontonoz P. 2010. Liver X receptor signaling pathways and atherosclerosis. Arterioscler. Thromb. Vasc. Biol. 30, 1513-1518.

Chen W, Wang N, Tall AR. 2005. PEST deletion mutant of ABCA1 shows impaired internalization and defective cholesterol efflux from late endosomes. J. Biol. Chem. 280, 29277-29281.

Chen J, Jiang Y, Liang Y, Tian X, Peng C, Ma KY, Liu J, Huang $Y$, Chen ZY. 2012. DPA n-3, DPA n-6 and DHA improve lipoprotein profiles and aortic function in hamsters fed a high cholesterol diet. Atherosclerosis. 221, 397-404.

Choi JS, Bae JY, Kim DS, Li J, Kim JL, Lee YJ, Kang YH. 2010. Dietary compound quercitrin dampens VEGF induction and PPARgamma activation in oxidized LDL-exposed murine macrophages: association with scavenger receptor CD36. 58, 1333-1341.

Clark SB, Tercyak AM. 1984. Reduced cholesterol transmucosal transport in rats with inhibited mucosal acyl CoA:cholesterol acyltransferase and normal pancreatic function. J. Lipid Res. 25, 148-159.

Damm EM, Pelkmans L, Kartenbeck J, Mezzacasa A, Kurzchalia T, Helenius A. 2005. Clathrin- and caveolin-1-independent endocytosis: entry of simian virus 40 into cells devoid of caveolae. J. Cell Biol. 168, 477-488.

Dietschy JM, Turley SD, Spady DK. 1993. Role of liver in the maintenance of cholesterol and of cholesterol and low density lipoprotein homeostasis in different animal species, including humans. J. Lipid Res. 34, 1637-1659

Dong P, Flores J, Pelton K, Solomon KR. 2010. Prohibitin is a cholesterol-sensitive regulator of cell cycle transit. .J Cell Biochem. 111, 1367-1374.

Farese RV Jr, Walther TC. 2009. Lipid droplets finally get a little R-E-S-P-E-C-T. Cell. 139, 855-860.

Feng B, Yao PM, Li Y, Devlin CM, Zhang D, Harding HP, Sweeney M, Rong JX, Kuriakose G, Fisher EA, Marks AR, Ron D, Tabas I. 2003. The endoplasmic reticulum is the site of cholesterol-induced cytotoxicity in macrophages. Nat. Cell Biol. 5, 781-792.

Fielding CJ, Bist A, Fielding PE. 1997. Caveolin mRNA levels are up-regulated by free cholesterol and downregulated by oxysterols in fibroblast monolayers. Proc. Natl. Acad. Sci. USA. 94, 3753-3758.

Fitzgerald ML, Mujawar Z, Tamehiro N. 2010. ABC transporters, atherosclerosis and inflammation. Atherosclerosis. 211, 361-370.

Girard E, Paul JL, Fournier N, Beaune P, Johannes L, Lamaze C, Védie B. 2011. The Dynamin Chemical Inhibitor Dynasore Impairs Cholesterol Trafficking and Sterol-Sensitive Genes Transcription in Human HeLa Cells and Macrophages. PLoS One. 6, e29042.

Goldstein JL, DeBose-Boyd RA, Brown MS. 2006. Protein sensors for membrane sterols. Cell. 124, 35-46.

Gong Y, Lee JN, Lee PC, Goldstein JL, Brown MS, Ye J. 2006. Sterol-regulated ubiquitination and degradation of Insig-1 creates a convergent mechanism for feedback control of cholesterol synthesis and uptake. Cell Metab. 3, 15-24.

Hanada K, Kumagai K, Yasuda S, Miura Y, Kawano M, Fukasawa M, Nishijima M. 2003. Molecular machinery for non-vesicular trafficking of ceramide. Nature. 426, 803-809. 
Hannan LA, Edidin M. 1996. Traffic, polarity, and detergent solubility of a glycosylphosphatidylinositol-anchored protein after LDL-deprivation of MDCK cells. .J Cell Biol. 133, 1265-1276.

Hassan HH, Denis M, Lee DY, latan I, Nyholt D, Ruel I, Krimbou L, Genest J. 2007. Identification of an ABCA1-dependent phospholipid-rich plasma membrane apolipoprotein A-I binding site for nascent HDL formation: implications for current models of HDL biogenesis. J. Lipid Res. 48, 2428-2442.

Hauet T, Liu J, Li H, Gazouli M, Culty M, Papadopoulos V. 2002. PBR, StAR, and PKA: partners in cholesterol transport in steroidogenic cells. Endocr. Res. 28, 395-401.

Howles PN, Carter CP, Hui DY. 1996. Dietary free and esterified cholesterol absorption in cholesterol esterase (bile salt-stimulated lipase) gene-targeted mice. J. Biol. Chem. 271, 7196-7202.

Ioannou YA. 2001. Multidrug permeases and subcellular cholesterol transport. Nat. Rev. Mol. Cell Biol. 2, 657668.

Ikonen E. 2008. Cellular cholesterol trafficking and compartmentalization. Nat. Rev. Mol. Cell Biol. 9, 125138.

Iqbal J, Hussain MM. Intestinal lipid absorption. 2009. Am. J. Physiol. Endocrinol. Metab. 296, E1183E1194,

Jakulj L, Vissers MN, Tanck MW, Hutten BA, Stellaard F, Kastelein JJ, Dallinga-Thie GM. 2010. ABCG5/G8 polymorphisms and markers of cholesterol metabolism: systematic review and meta-analysis. J. Lipid Res. 51, 3016-3023.

Janowski BA, Grogan MJ, Jones SA, Wisely GB, Kliewer SA, Corey EJ, Mangelsdorf DJ. 1999. Structural requirements of ligands for the oxysterol liver $\mathrm{X}$ receptors LXRalpha and LXRbeta. Proc. Natl. Acad. Sci. USA. 96, 266-271.

Jaureguiberry MS, Tricerri MA, Sanchez SA, Garda HA, Finarelli GS, Gonzalez MC, Rimoldi OJ. 2010. Membrane organization and regulation of cellular cholesterol homeostasis. J. Membr. Biol. 234, 183-194.

Jefcoate C. 2002. High-flux mitochondrial cholesterol trafficking, a specialized function of the adrenal cortex. J. Clin. Invest. 110, 881-890.

Kamisako T, Tanaka Y, Ikeda T, Yamamoto K, Ogawa H. 2012. Dietary fish oil regulates gene expression of cholesterol and bile acid transporters in mice. Hepatol. Res. 42, 321-326.

Kim A, Chiu A, Barone MK, Avino D, Wang F, Coleman Cl, Phung OJ. 2011. Green tea catechins decrease total and low-density lipoprotein cholesterol: a systematic review and meta-analysis. J. Am. Diet Assoc. 111, 1720-1729.

Kotani K, Fujiwara S, Tsuzaki K, Sano Y, Nagai N, Yamada T, Sakane N. 2011. The Association Between the Uncoupling Protein-1 Gene A-3826G Polymorphism and High-density Lipoprotein Cholesterol in A General Japanese Population: A Consideration of the Obesity Status. J. Clin. Med. Res. 3, 319-324.

Kuiper GG, Lemmen JG, Carlsson B, Corton JC, Safe $\mathrm{SH}$, van der Saag PT, van der Burg B, Gustafsson JA 1998. Interaction of estrogenic chemicals and phytoestrogens with estrogen receptor $\beta$. Endocrino$\log y 139,4252-4263$.

Laatsch A, Panteli M, Sornsakrin M, Hoffzimmer B, Grewal T, Heeren J. 2012. Low density lipoprotein receptor-related protein 1 dependent endosomal trapping and recycling of apolipoprotein E. PLoS One. 7, e29385.
Lewis G.F. 1997. Fatty acid regulation of very low density lipoprotein production. Curr. Opin. Lipidol. 8, 146-153.

Lichtenstein L, Berbée JF, van Dijk SJ, van Dijk KW, Bensadoun A, Kema IP, Voshol PJ, Müller M, Rensen PC, Kersten S. 2007. Angptl4 upregulates cholesterol synthesis in liver via inhibition of LPL- and HL-dependent hepatic cholesterol uptake. Arterioscler. Thromb. Vasc. Biol. 27, 2420-2427.

Lin SX, Mallet WG, Huang AY, Maxfield FR. 2004. Endocytosed cation-independent mannose 6-phosphate receptor traffics via the endocytic recycling compartment en route to the trans-Golgi network and a subpopulation of late endosomes. Mol. Biol. Cell. 15, 721-733.

Liu J, Li H, Papadopoulos V. 2003. PAP7, a PBR/PKARlalpha-associated protein: a new element in the relay of the hormonal induction of steroidogenesis. J. Steroid Biochem. Mol. Biol. 85, 275-283.

López-Soldado I, Avella M, Botham KM. 2007. Comparison of the effects of dietary saturated, mono-unsaturated and polyunsaturated fatty acids on very-lowdensity lipoprotein secretion when delivered to hepatocytes in chylomicron remnant-like particles. Biochem. Soc. Trans. 35, 440-441.

López-Soldado I, Avella M, Botham KM. 2009. Differential influence of different dietary fatty acids on very low-density lipoprotein secretion when delivered to hepatocytes in chylomicron remnants. Metabolism. 58, 186-195.

Lordan S, Mackrill JJ, O’Brien NM. 2009. Oxysterols and mechanisms of apoptotic signaling: implications in the pathology of degenerative diseases. J. Nutr. Biochem. 20, 321-336.

Magyar K, Halmosi R, Palfi A, Feher G, Czopf L, Fulop A, Battyany I, Sumegi B, Toth K, Szabados E. 2012. Cardioprotection by resveratrol: A human clinical trial in patients with stable coronary artery disease. Clin. Hemorheol. Microcirc. 50, 179-187.

Martínez-Seara H, Róg T, Karttunen M, Vattulainen I, Reigada R. 2010. Cholesterol induces specific spatial and orientational order in cholesterol/phospholipid membranes. PLoS One. 5, e11162.

Martini C, Pallottini V. 2007. Cholesterol: from feeding to gene regulation. Genes Nutr. 2, 181-193.

Mathews SA, Oliver WT, Phillips OT, Odle J, DiersenSchade DA, Harrell RJ. 2002. Comparison of triglycerides and phospholipids as supplemental sources of dietary long-chain polyunsaturated fatty acids in piglets. J. Nutr. 132, 3081-2089.

Medina MW, Gao F, Naidoo D, Rudel LL, Temel RE, McDaniel AL, Marshall SM, Krauss RM. 2011. Coordinately regulated alternative splicing of genes involved in cholesterol biosynthesis and uptake. PLoS One. 6, e19420.

Meurs I, Van Eck M, Van Berkel TJ. 2010. High-density lipoprotein: key molecule in cholesterol efflux and the prevention of atherosclerosis. Curr. Pharm. Des. 16, 1445-1467.

Mukherjee S, Zha X, Tabas I, Maxfield FR. 1998. Cholesterol distribution in living cells: fluorescence imaging using dehydroergosterol as a fluorescent cholesterol analog. Biophys. J. 75, 1915-1925.

Nabi IR, Le PU. 2003. Caveolae/raft-dependent endocytosis. J. Cell Biol. 161, 673-677.

Nickel W, Brügger B, Wieland FT. 1998. Protein and lipid sorting between the endoplasmic reticulum and the Golgi complex. Semin. Cell De.v Biol. 9, 493-501.

Okuhira K, Fitzgerald ML, Sarracino DA, Manning JJ, Bell SA, Goss JL, Freeman MW. 2005. Purification of ATPbinding cassette transporter $\mathrm{A} 1$ and associated binding 
proteins reveals the importance of beta1-syntrophin in cholesterol efflux. J. Biol. Chem. 280, 39653-39664.

Okuhira K, Fitzgerald ML, Tamehiro N, Ohoka N, Suzuki K, Sawada J, Naito M, Nishimaki-Mogami T. 2010. Binding of PDZ-RhoGEF to ATP-binding cassette transporter A1 (ABCA1) induces cholesterol efflux through RhoA activation and prevention of transporter degradation. J. Biol. Chem. 285, 16369-16377.

Olofsson SO, Boström P, Andersson L, Rutberg M, Perman J, Borén J. 2009. Lipid droplets as dynamic organelles connecting storage and efflux of lipids. Biochim. Biophys. Acta. 1791, 448-458.

Olson RE. 1998. Discovery of the lipoproteins, their role in fat transport and their significance as risk factors. $J$. Nutr. 128 (2 Suppl).439S-443S.

Ortegren U, Karlsson M, Blazic N, Blomqvist M, Nystrom FH, Gustavsson J, Fredman P, Strålfors P. 2004. Lipids and glycosphingolipids in caveolae and surrounding plasma membrane of primary rat adipocytes. Eur. J. Biochem. 271, 2028-2036.

Otsuka J. 2009. A theoretical approach to the large-scale evolution of multicellularity and cell differentiation. J. Theor. Biol. 255, 129-136.

Pan SL, Wang F, Lu ZP, Liu CW, Hu CY, Luo H, Peng JH, Luo XQ, Pang GF, Lu SH, Wu HY, Huang LJ, Yin RX. 2012. Cholesteryl ester transfer protein TaqlB polymorphism and its association with serum lipid levels and longevity in Chinese Bama Zhuang population. Lipids Health Dis. 15, 11-26.

Pilch PF, Meshulam T, Ding S, Liu L. 2011. Caveolae and lipid trafficking in adipocytes. Clin. Lipidol. 6, 49-58.

Rohlmann A, Gotthardt M, Hammer RE, Herz J. 1998. Inducible inactivation of hepatic LRP gene by cre-mediated recombination confirms role of LRP in clearance of chylomicron remnants. J. Clin. Invest. 101, 689-695.

Rone MB, Fan J, Papadopoulos V. 2009. Cholesterol transport in steroid biosynthesis: role of protein-protein interactions and implications in disease states. Biochim. Biophys. Acta. 1791, 646-658.

Ronis MJ, Chen Y, Badeaux J, Badger TM. 2009. Dietary soy protein isolate attenuates metabolic syndrome in rats via effects on PPAR, LXR, and SREBP signaling. J. Nutr. 139, 1431-1438.

Rothberg KG, Heuser JE, Donzell WC, Ying YS, Glenney JR, Anderson RG. 1992. Caveolin, a protein component of caveolae membrane coats. Cell. 68, 673-682.

Santamarina-Fojo S, Dugi KA. 1994. Structure, function and role of lipoprotein lipase in lipoprotein metabolism. Curr. Opin. Lipidol. 5, 117-125.

Sato R. 2010. Sterol metabolism and SREBP activation. Arch. Biochem. Biophys. 501, 177-181.

Shaul PW. 2003. Endothelial nitric oxide synthase, caveolae and the development of atherosclerosis. J. Physiol. 547, 21-33.
Simons K, Vaz WL. 2004. Model systems, lipid rafts, and cell membranes. Annu. Rev. Biophys. Biomol. Struct. 33, 269-295.

Smith JD. 2010. Apolipoprotein A-I and its mimetics for the treatment of atherosclerosis. Curr Opin Investig Drugs. 11, 989-996.

Storch J, Xu Z. 2009. Niemann-Pick C2 (NPC2) and intracellular cholesterol trafficking. Biochim. Biophys. Acta. 1791, 671-678.

Sukonina V, Lookene A, Olivecrona T, Olivecrona G. 2006. Angiopoietin-like protein 4 converts lipoprotein lipase to inactive monomers and modulates lipase activity in adipose tissue. Proc. Natl. Acad. Sci. USA. 103, 17450-17455.

Tamehiro N, Zhou S, Okuhira K, Benita Y, Brown CE, Zhuang DZ, Latz E, Hornemann T, von Eckardstein A, Xavier RJ, Freeman MW, Fitzgerald ML. 2008. SPTLC1 binds ABCA1 to negatively regulate trafficking and cholesterol efflux activity of the transporter. Biochemistry. 47, 6138-6147.

Thorn H, Stenkula KG, Karlsson M, Ortegren U, Nystrom FH, Gustavsson J, Stralfors P. 2003. Cell surface orifices of caveolae and localization of caveolin to the necks of caveolae in adipocytes. Mol. Biol. Cell. 14, 39673976.

Tontonoz P, Mangelsdorf DJ. 2003. Liver X receptor signaling pathways in cardiovascular disease. Mol. Endocrinol. 17, 985-993.

Valenzuela A, Sanhueza, J. 2009. Adipose tissue: something more than a reservoir of energy. Grasas Aceites. 60, 439-452.

van der Velde AE, Brufau G, Groen AK. 2010. Transintestinal cholesterol efflux. Curr. Opin. Lipidol. 21, 167-171.

Waugh MG, Chu KM, Clayton EL, Minogue S, Hsuan JJ. 2011. Detergent-free isolation and characterization of cholesterol-rich membrane domains from trans-Golgi network vesicles. J. Lipid Res. 52, 582-589.

Weber LW, Boll M, Stampfl A. 2004. Maintaining cholesterol homeostasis: sterol regulatory element-binding proteins. World J. Gastroenterol. 10, 3081-3087

Weinstein MM, Yin L, Tu Y, Wang X, Wu X, Castellani LW, Walzem RL, Lusis AJ, Fong LG, Beigneux AP, Young SG. 2010. Chylomicronemia elicits atherosclerosis in mice brief report. Arterioscler. Thromb. Vasc. Biol. 30, 20-23.

Yao PM, Tabas I. 2000. Free cholesterol loading of macrophages induces apoptosis involving the fas pathway. J. Biol. Chem. 275, 23807-23813.

Zhao C, Dahlman-Wright K. 2010. Liver X receptor in cholesterol metabolism. J. Endocrinol. 204, 233-240.

Recibido: 22/3/12 Aceptado: $31 / 5 / 12$ 